\title{
A structure-based Multiple-Instance Learning approach to predicting in vitro transcription factor-DNA interaction
}

\author{
Zhen Gao*, Jianhua Ruan* \\ From IEEE International Workshop on Genomics Signal Processing and Statistics (GENSIPS) 2013 \\ Houston,TX, USA. 17-19 November 2013
}

\begin{abstract}
Background: Understanding the mechanism of transcriptional regulation remains an inspiring stage of molecular biology. Recently, in vitro protein-binding microarray experiments have greatly improved the understanding of transcription factor-DNA interaction. We present a method - MIL3D - which predicts in vitro transcription factor binding by multiple-instance learning with structural properties of DNA.

Results: Evaluation on in vitro data of twenty mouse transcription factors shows that our method outperforms a method based on simple-instance learning with DNA structural properties, and the widely used k-mer counting method, for nineteen out of twenty of the transcription factors. Our analysis showed that the MIL3D approach can utilize subtle structural similarities when a strong sequence consensus is not available.
\end{abstract}

Conclusion: Combining multiple-instance learning and structural properties of DNA has promising potential for studying biological regulatory networks.

\section{Introduction}

Modeling of transcription factor binding sites (TFBS), sometimes also referred to as transcription factor binding motifs, is a crucial step towards understanding molecular regulatory networks. Among the popular methods for modeling TFBS, position-specific weight matrix (PWM) $[1,2]$ and $k$-mer based approaches $[3,4]$ have gained great success $[5,6]$. Providing a probability score for each of the four nucleotide bases of each position of a TFBS, PWMbased approaches are intuitive for representing the sequence preferences of a transcription factor (TF), and easy to visualize for the TFBS models. However, these approaches have some limitations, including inefficiency in optimizing PWM, difficulty to represent some TFBSs (such as structural motifs and those with variable lengths) by a single PWM, and incapability to capture positional dependency $[3,4,7,8]$. In contrast, $k$-mer based approaches use a word-based sequence of length $k$ to

\footnotetext{
* Correspondence: zhen.gao@utsa.edu; jianhua.ruan@utsa.edu Department of Computer Science, The University of Texas at San Antonio, One UTSA Circle, San Antonio, TX 78249, USA
}

enumerate possible instances of a motif. Compared to PWM-based approaches, the $k$-mer based approaches can be designed to make fewer assumptions about the degree of TFBS degeneracy, length of the binding sites, and position dependences of a motif. The $k$-mer based approaches have been found to provide more accurate models than PWMs in general $[5,6,9]$. But these models usually involve too many parameters and may not unravel the underlying knowledge of TF-DNA interactions.

A TF binding site is usually 8 to 12 -mer base pair, determined by the sequence specificity and its physicochemical properties (structural and chemical properties), which are overlooked by both the PWM based and the $k$ mer based approaches. In [10], a novel TFBS modeling and predicting approach is presented, where the sequence-specific chemical and structural features of DNA are applied. Based on their evaluation using an in vivo ChIP-chip dataset, their method outperforms four previous methods [1,11-13] by reporting fewer false positive matches. Their method provides a new perspective for understanding TF-DNA interactions. On the other 
hand, the in vivo protein-DNA interactions observed in ChIP-chip assays are not necessarily direct [14], as some TFs tend to interact with DNA extensively through other partners. Therefore, an evaluation on a proper in vitro dataset would be more appropriate to reveal the benefit of such physicochemical features in modeling TF-DNA interactions.

Protein-binding microarray (PBM) is a high-throughput experiment used to measure the in vitro binding affinity of a given TF to the sequences on the probe array [15]. A typical design of the array consists of an exhaustive enumeration of all possible 10-mers concatenated into $\sim 40,000$ unique probe nucleic acid sequences, each containing 35 bases. The PBM score represents the relative binding affinity of a given TF to each probe sequence on the array. Because typical confounding factors such as transcription cofactors present in ChIP-based experiments are eliminated, PBM data provides an excellent information source to develop physicochemical models for TF-DNA interactions. On the other hand, the currently available physicochemical features are mainly 3-mer or 4-mer based [10]. Direct mapping of the 3-mer or 4mer based meta-features to the candidate DNA binding sequences as in the work of [10] may not reflect the TF-DNA binding nature, since a TFBS usually spans 6 to 12 base pairs (bp), and its exact location within the PBM probe sequence is unknown. As a result, conventional machine learning algorithms, which rely on well-structured instance (feature vector) and label pairs, may not work well in modeling PBM data.

In this paper we propose a novel approach, MIL3D, to predict in vitro transcription factor binding based on the structural properties of DNA using the so-called multipleinstance learning (MIL), which was originally developed in the mid ' 90 s to deal with uncertainty in instance labels and has found many interesting applications in bioinformatics and information retrieval [16-19]. In a conventional classification problem, the input is a set of instances (feature vectors), each of which is labeled positive or negative. In contrast, the input of MIL is a set of bags that can have many instances, but the instances are not individually labeled - instead, the labels are associated with bags. The common assumption is that a bag with a positive label contains at least one positive instance (whose identity is unknown), while all instances in the negative bag should have negative labels. The MIL framework fits the TFBS modeling scenario very well, because it is commonly assumed that a DNA sequence that can be bound by a TF (positive sequence) should contain one or more TFBSs, while a DNA sequence that cannot be bound by the TF (negative sequence) should has no TFBS. The exact location of the TFBS within the positive sequence is typically not fixed, although some preference might exist.
Therefore, it is fairly intuitive to consider each DNA sequence as a bag, and any subsequence that can be a potential binding site as an instance.

In our algorithm, we treat each PBM probe sequence as a bag, and label the bags positive or negative according to the affinity level from the TF binding data. Each PBM probe is then decomposed into a set of $k$-mers using a simple sliding-window approach, and each $k$-mer is represented by a vector describing its structural properties. We then apply an existing MIL algorithm to learn a classification model that can correct predict the labels for the bags.

To demonstrate the advantage of our approach, we compared the performance of our algorithm with the conventional single-instance based learning (SIL), and with the simple $k$-mer counting method. Experimental results on PBM data of twenty mouse TFs showed that our method outperformed those methods with significant margins. On the other hand, it is also worth noting that our goal in this current paper is not a state-of-the-art method to predict TF-DNA interaction with the highest accuracy. To our knowledge, this is the first work that demonstrates the feasibility of using the MIL paradigm and structural properties in modeling TF-DNA interactions. We believe that many of the existing $k$-mer based methods for predicting TF-DNA interactions, which often involve filtering, normalization, and transformation of the binding data, can be combined with the key idea proposed in this paper to obtain a more accurate model.

\section{Materials and methods}

\section{The in vitro transcription factor-DNA binding data}

The protein binding microarray (PBM) data is acquired from [5]. Two completely different array designs, each of which consists of 40,000 unique 35-mers, are used for twenty different mouse TFs. In the arrays, all possible 10mers, and 32 copies of every non-palindromic 8-mer are included, presenting an unbiased study of TF binding preferences. The data were used for training in the Dialogue on Reverse-Engineering Assessment and Methods (DREAM) competition [20] and the data is freely available on the DREAM5 competition website upon registration. In our experiment, we selected 3000 probes with the highest binding signals as positive sequences and 3000 probes with the lowest binding signals as negative for each of the twenty TFs. We used the two different sets of arrays for training and testing respectively. As the training and testing data are completely separate, and actually come from different sources, this setting makes the prediction problem more challenging and the results less likely to be influenced by biases, compared to evaluations based on, for example, 10-fold cross-validation, where training and testing data could share some unknown similarities and bias the evaluation results. 


\section{MIL model for TF-DNA binding}

Conventional classification algorithms deal with instances that consist of feature vector-label pairs, where each instance (e.g., probe sequence) is represented by a wellstructured feature vector and has a label. The modeling task is to extract useful information (e.g., a subset of features) to map to the labels. A crucial difference between MIL and conventional learning algorithms is that in MIL, labels are associated with bags (as opposed to instances), and each bag can contain multiple instances. While the instances in the same bag do not have their own labels, depending on the actual MIL algorithm implementation, it is assumed that they will contribute to the label of the bag in a binary or probabilistic way. In modeling PBM data, it is reasonable to assume that a probe sequence with high binding affinity (positive sequence) contains one or more binding sites, while a probe sequence with low affinity (negative sequence) does not have any binding sites. Therefore, we consider each candidate binding site ( $k$-mer, $k \in[5,8]$ in this study) as an instance and all possible $k$-mers in a probe sequence as a bag. Bags are labeled positive or negative according to the binding affinity of the probe sequences as mentioned above. Each instance is mapped to a single feature vector representing the structural properties of the $k$-mer, as will be discussed later (Figure 1).

To apply MIL to model the transcription factor binding site, we use the multiple-instance-wrapper algorithm [21] implemented in the WEKA machine learning package [22], and used the popular C4.5 decision tree algorithm (named J48 in WEKA) as the base single-instance classifier. The choice of C4.5 is because it is a simple and less biased classifier - it does not need to adjust a parameter to optimize its performance, and the number of features of an instance has little influence on its performance. Moreover, the difference of range and scale among each feature has little influence on the performance as well.

The wrapper-based MIL approach enables us to compare MIL-based TF-DNA interaction models and singleinstance based model in a relatively unbiased manner, because the wrapper-based method can use any traditional single-instance classifiers as a base classifier. In contrast, a non-wrapper based MIL algorithm does not use SIL classifiers directly, which makes it hard to underpin the source of the performance difference between the two. The wrapped approach is derived from the assumption that a bag's label is formed by the overall contribution of all the instances within the bag, which is also consistent with the understanding that multiple binding sites may contribute to recruiting TF additively. Based on the above assumption, at the learning time, the wrapper algorithm converts the MIL training and testing data to single-instance based learning data by giving each instance a label that is the same as its bag, and each instance is initially assigned a weight proportional to the inverse of the size of the bag that they belong to. In our case, as every bag has the same size (35-k+1 instances each representing a $k$-mer), the initial weight for every instance is $\frac{1}{35-k+1}$. This setting is because we do not have any prior knowledge for the location of the TFBS within the probe sequence, and therefore we assume that all $k$-mers within the 35 -mer have the same probability being the real binding site, which will be updated iteratively when the model becomes more accurate. At prediction time, the class probability for a bag is estimated by averaging the class probabilities assigned to the individual instances in the bag. Moreover, the class probability for each individual instance can also be extracted from the positive bags to identify possible binding site(s) for each probe sequence. As the result of the above designs, different from most of the other nonwrapper based MIL classifiers, the wrapper algorithm can be combined with any traditional single-instance classifiers, which enables an unbiased comparison between our MIL-based TFBS modeling method with the traditional SIL based TFBS modeling method.

The choice of decision tree rather than other popular algorithms such as support vector machines (SVM) is because of the relative parameter-free robust performance and the implicit feature selection provided by the former method, which makes it ideal for evaluating and comparing the performance of the MIL-based and SIL-based approaches. For example, the performance of SVM approach is very sensitive to kernel choices and other parameters including the soft margin parameter and feature normalization method. As the $k$-mer based approaches and our structure-based MIL approach have very different number of features and different feature characteristics, (e.g., in $k$-mer based approach, all features are small integers, while in our method the features are typically real values and different features can have different distributions), we feel a simple base classifier such as a decision tree can provide a more fair comparison.

\section{The meta-features of DNA structural properties}

The structural properties of DNA is obtained from [10], which describe the steric and conformational rigidity properties of DNA. The structural features of DNA oligomers were defined based on the predicted average 3-dimensional structures of short DNA sequences, which were determined via MD simulations [23]. The structural feature table of 3-mer is a 64 by 6 matrix, which contains six real value "base" parameters for each middle base pair of all the $64\left(4^{3}\right)$ different 3 -mers. The base parameters measure the relative relations between two fundamental units of the middle based pair of a 3-mer in a 3-dimensional space. The six base parameters 


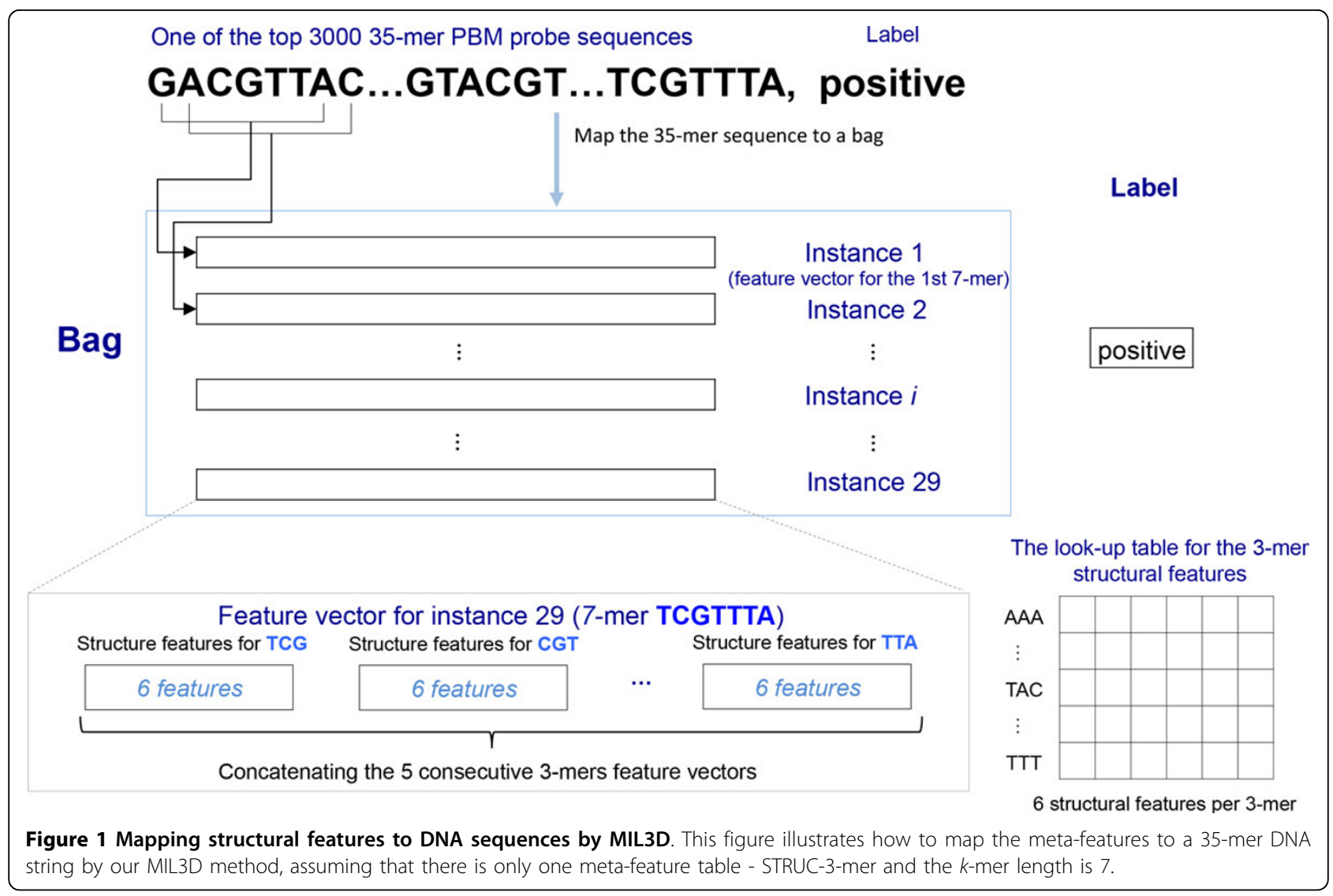

are shear, buckle, stretch, propeller, stagger and opening [24].

\section{Mapping PBM sequences to feature vectors}

Figure 1 shows the steps of mapping the 35-mer PBM probe sequence to the structural feature vectors by our MIL3D method. In this example the $k$-mer length is specified as 7. As mentioned before, each probe sequence is treated as a bag and has a negative or positive label depending on its binding affinity to a particular TF. Each of the 29 shifted 7-mers in the 35-mer is treated as an instance, which does not have a label itself. For each instance (7-mer), we obtain the structural features of each of the 5 triplets using the structural feature table mentioned above as a lookup table. The feature vectors for the 5 triplets from the same 7-mer are then concatenated, in the same order as they appear in the 7-mer, to form a single feature vector for the 7-mer, which is an instance in the MIL model.

Table 1 shows the detailed profiles of the MIL3D method as well as the comparison approaches, including a SIL model with the structural meta-features - SIL3D [10], and the simple traditional $k$-mer based approaches. In SIL3D, each 35-mer is treated as an instance, whose feature vector is simply the concatenation of the structure features of the 33 continuous 3 -mers from the 35-mer, and SIL instead of MIL is applied to learn the classification model. The $k$-mer based approach also uses SIL. It counts the number of occurrences for every possible $k$-mer and uses the $k$-mer counts as the feature vector in the model. We have tried $k$-mer length from 5 to 8 for the MIL3D method and $k$-mer length from 3 to 8 for the simple counting method. In addition, for the $k$-mer based method, we included a ' $3+4+5$ mer counting' model, which uses counts of all 3-, 4-and 5-mers as features. The comparison between MIL3D and SIL3D will show the benefit of using MIL, while the comparison between MIL3D and the $k$-mer based methods is expected to show the benefit from using the structural features and MIL.

\section{Results}

The predicting Area Under ROC (AUC) scores for each model-TF pair are given in Figure 2. As shown, SIL3D consistently has the worst performance, which is as expected. This is because in SIL3D, the 3-mer based feature vectors are concatenated by the order they appear in the 35-mer, and therefore bears the implicit assumption that the same position different probe sequences have the same contribution in determining TF binding affinity, which is in contrary to the common knowledge that the 
Table 1 Summary of different feature models.

\begin{tabular}{|c|c|c|c|c|}
\hline Name & $\begin{array}{l}\text { Length of } \\
k \text {-mer } \\
\text { kernel }\end{array}$ & $\begin{array}{l}\text { Number of } \\
\text { instances per } \\
\text { bag }\end{array}$ & $\begin{array}{l}\text { Number of features per } \\
\text { instance }\end{array}$ & Description \\
\hline MIL3D_kmer & $k \in[5,8]$ & $35-k+1$ & $\begin{array}{l}k-3+1 \text { triplets per } k \text {-mer } * 6 \text { base } \\
\text { structural features per triplet }\end{array}$ & $\begin{array}{l}\text { For each of the } 35-k+1 \text { different continuous } k \text {-mers in the } 35 \text {-mer, } \\
\text { for each of the } k-3+1 \text { triplets, map the structural features to the } \\
k \text {-mer sequentially. The feature vector of one } k \text {-mer represent an } \\
\text { instance, and the } 35-k+1 \text { instances form a bag. }\end{array}$ \\
\hline SIL3D & 3 & 1 & $\begin{array}{l}198 \text { (6 structural features per } \\
\text { triplet * } 33 \text { continous } 3 \text {-mers in } \\
\text { the } 35 \text {-mer) }\end{array}$ & $\begin{array}{l}\text { For each of the } 33 \text { different continous } 3 \text {-mers in the } 35 \text {-mer, map } \\
\text { the } 6 \text { structural features to the } 3 \text {-mer. }\end{array}$ \\
\hline kmer_counting & $k \in[3,8]$ & 1 & $\begin{array}{l}4^{k} \text { (number of occurrences of all } \\
\text { different } k \text {-mers) }\end{array}$ & $\begin{array}{l}\text { For each } 35 \text {-mer DNA sequence in the PBM array, count the } \\
\text { number of occurrences for each of the } 4^{k} k \text {-mers; map the } k \text {-mer } \\
\text { counter table to the PBM sequence. This } k \text {-mer based method has } \\
\text { been widely used in the previous decades and has been proven } \\
\text { to be still very effective at present [5]. }\end{array}$ \\
\hline $\begin{array}{c}3+4 \\
+5 \text { mer_counting }\end{array}$ & 3,4 and 5 & 1 & $1344(64+256+1024)$ & $\begin{array}{l}\text { For each } 35 \text {-mer DNA sequence in the PBM array, map the above } \\
3 \text { counter tables (including 3-mer, 4-mer and 5-mer tables) to the } \\
\text { sequence. }\end{array}$ \\
\hline
\end{tabular}

TF-DNA interaction is relatively independent of the position of the binding site. In contrast, MIL3D does not explicitly consider the position of the 3 -mers in the probe sequence, but instead attempts to find the most contributing instance in a bag. In other words, MIL helps find the real TFBSs within a PBM probe sequence.

The MIL3D method performs significantly better than the traditional $k$-mer counting method. Furthermore, changing of instance $(k$-mer) length in the MIL3D model has almost no impact on the performance while the performance of $k$-mer based method significantly depends on the value of $k$. The best performance of the $k$-mer based method is with $k$ equal to 5 . Larger values of $k$ cause a significant degradation of the performance of the $k$-mer based method, presumably due to overfitting associated with the exponentially growing number of features and sparsity of the feature vectors when $k$ increases. For example, each instance of the 7-mer counting has 16384 features while the total number of instances in our experiment is only 6000. In contrast, the number of features in MIL3D is linear to the number of 3-mers contained in the $k$-mer, and therefore is relatively stable when the value of $k$ changes. While the actual TFBS length might be different from $k$, the MIL3D model can overcome the problem by modeling the TFBS with multiple overlapping instances, each of which can be a partial binding site.

As shown in Figure 2, MIL3D models are consistently better than $k$-mer based models for almost all TFs, often by a significant margin, except for Tcf3, where the 3+4+5-mer counting approach works slightly better than MIL3D models. The average AUC for MIL3D (0.94) is significantly

\begin{tabular}{|c|c|c|c|c|c|c|c|c|c|c|c|c|c|}
\hline TF_NO & TF_Name & $\underset{\text { mer }}{\text { MIL3D_6 }}$ & $\underset{\text { mer }}{\text { MIL3D_5 }}$ & $\underset{\text { mer }}{\text { MIL3D_8 }}$ & $\begin{array}{c}\text { MIL3D_7 } \\
\text { mer }\end{array}$ & $\begin{array}{l}\text { 5mer_} \\
\text { counting }\end{array}$ & $\begin{array}{l}6 \text { mer_ } \\
\text { counting }\end{array}$ & $\begin{array}{l}3+4+5 \mathrm{mer} \\
\text { _counting }\end{array}$ & $\begin{array}{l}\text { 4mer__} \\
\text { counting }\end{array}$ & $\begin{array}{l}\text { 3mer__} \\
\text { counting }\end{array}$ & SIL3D & $\begin{array}{l}7 \text { mer_- } \\
\text { counting }\end{array}$ & $\begin{array}{l}\text { 8mer__ } \\
\text { counting }\end{array}$ \\
\hline 1 & Cebpb & 0.983 & 0.983 & 0.983 & 0.985 & 0.906 & 0.891 & 0.857 & 0.840 & 0.841 & 0.710 & 0.668 & 0.506 \\
\hline 2 & Esr1 & 0.842 & 0.832 & 0.823 & 0.837 & 0.810 & 0.792 & 0.806 & 0.783 & 0.702 & 0.600 & 0.714 & 0.519 \\
\hline 3 & Foxo1 & 0.976 & 0.972 & 0.975 & 0.977 & 0.886 & 0.898 & 0.823 & 0.820 & 0.813 & 0.668 & 0.753 & 0.500 \\
\hline 4 & Foxo4 & 0.991 & 0.990 & 0.992 & 0.993 & 0.962 & 0.944 & 0.933 & 0.919 & 0.893 & 0.757 & 0.856 & 0.503 \\
\hline 5 & Foxp2 & 0.953 & 0.955 & 0.947 & 0.950 & 0.779 & 0.770 & 0.799 & 0.781 & 0.815 & 0.687 & 0.626 & 0.502 \\
\hline 6 & Irf2 & 0.979 & 0.975 & 0.981 & 0.978 & 0.922 & 0.905 & 0.907 & 0.889 & 0.896 & 0.817 & 0.718 & 0.500 \\
\hline 7 & Mecp2 & 0.992 & 0.992 & 0.992 & 0.992 & 0.886 & 0.867 & 0.885 & 0.865 & 0.890 & 0.757 & 0.596 & 0.500 \\
\hline 8 & Pou3f1 & 0.976 & 0.973 & 0.977 & 0.977 & 0.899 & 0.905 & 0.845 & 0.866 & 0.848 & 0.763 & 0.664 & 0.500 \\
\hline 9 & Sp1 & 0.929 & 0.922 & 0.925 & 0.932 & 0.749 & 0.792 & 0.747 & 0.749 & 0.759 & 0.666 & 0.574 & 0.510 \\
\hline 10 & Tcf3 & 0.768 & 0.763 & 0.765 & 0.771 & 0.706 & 0.728 & 0.778 & 0.663 & 0.647 & 0.740 & 0.667 & 0.500 \\
\hline 11 & Egr2 & 0.940 & 0.930 & 0.950 & 0.952 & 0.896 & 0.893 & 0.856 & 0.826 & 0.777 & 0.636 & 0.784 & 0.576 \\
\hline 12 & Foxo3 & 0.988 & 0.989 & 0.986 & 0.989 & 0.868 & 0.837 & 0.847 & 0.856 & 0.844 & 0.732 & 0.712 & 0.500 \\
\hline 13 & Gmeb2 & 0.997 & 0.996 & 0.998 & 0.998 & 0.996 & 0.979 & 0.991 & 0.995 & 0.986 & 0.880 & 0.809 & 0.500 \\
\hline 14 & $\mathrm{Nr} 2 \mathrm{c} 1$ & 0.848 & 0.848 & 0.855 & 0.849 & 0.719 & 0.720 & 0.702 & 0.666 & 0.653 & 0.620 & 0.616 & 0.515 \\
\hline 15 & $\mathrm{~Tb} \times 3$ & 0.866 & 0.866 & 0.864 & 0.868 & 0.726 & 0.723 & 0.727 & 0.719 & 0.724 & 0.654 & 0.604 & 0.500 \\
\hline 16 & Foxj2 & 0.976 & 0.974 & 0.976 & 0.978 & 0.875 & 0.907 & 0.809 & 0.836 & 0.828 & 0.726 & 0.662 & 0.500 \\
\hline 17 & Foxp1 & 0.985 & 0.985 & 0.982 & 0.985 & 0.867 & 0.833 & 0.865 & 0.837 & 0.856 & 0.720 & 0.713 & 0.500 \\
\hline 18 & Junb & 0.955 & 0.958 & 0.939 & 0.948 & 0.762 & 0.784 & 0.741 & 0.756 & 0.800 & 0.747 & 0.596 & 0.500 \\
\hline 19 & Sox14 & 0.983 & 0.982 & 0.977 & 0.981 & 0.900 & 0.881 & 0.865 & 0.869 & 0.862 & 0.722 & 0.631 & 0.500 \\
\hline 20 & Zscan20 & 0.892 & 0.901 & 0.867 & 0.887 & 0.695 & 0.722 & 0.674 & 0.648 & 0.668 & 0.593 & 0.550 & 0.500 \\
\hline \multicolumn{2}{|c|}{ average } & 0.941 & 0.939 & 0.938 & 0.930 & 0.840 & 0.839 & 0.823 & 0.809 & 0.805 & 0.710 & 0.676 & 0.506 \\
\hline
\end{tabular}


higher than the average AUC for the 5 mer counting method $(0.84)$ ( $p$-value $=1 \mathrm{e}-7$, paired t-test). Among all MIL3D models, there are only five TFs out of twenty with AUCs below 0.91, while for the 5-mer counting models, there are only 5 TFs with AUCs above 0.90 . With the MIL3D method, there are 11 TFs with AUCs above 0.95, while with the 5-mer counting method, only has 2 TFs (Foxo4 and Gmeb2) have AUCs greater than 0.95. The performance of MIL3D on Esr1, Tcf3, Nr2C1 and Tbx3 are low compared to other TFs $(\mathrm{AUC}<0.86)$, despite being considerably better than the 5 -mer counting method, marking possible areas for improvement.

Figure 3 compares the performance of MIL3D 7-mer and 5 -mer counting on every TF. In the following result discussion, we use MIL3D to represent MIL3D 7-mer for short. The reason that we choose 7 as the main length of motif model is because 7 is one of the most common lengths of a motif core. It shows a direct performance comparison between the conventional 5mer-counting method and MIL3D, based on the AUC score. We can see that for the prediction of TF with the lowest score, such as Tcf3 (0.771 by MIL3D and 0.706 by 5 mer-counting), both methods do not perform well, while for some highest scoring TFs, such as Foxo4 and Gmeb2, the performance of the two methods also do not have much differences (all the AUCs for them is above 0.962). However, for the TFs with a relatively moderate predicting performance by 5 mer-counting, such as Sp1, Junb and Foxp2, the performance gained by
MIL3D is significant. Note that Junb binding preference is quite complex and shows non-canonical patterns, with multiple variable-length consensuses including TGA[G/C] TCA and TGACGT[C/T]A $[25,26]$. The AUC for Junb by 5 mer-counting is 0.762 , while it is 0.921 by MIL3D $(+0.159$ gain), which shows the advantage of MIL3D of modeling complex binding site. The AUC accuracy for Sp1 by 5 mer-counting is 0.749 , while by MIL3D it is 0.915 (+0.166 gain). The AUC for Foxp2 is 0.779 by 5 mer-counting, while it is 0.940 by MIL3D ( 0.161 gain).

To further investigate the fundamental reason for the improved prediction accuracy of MIL3D, we further analyzed the putative binding sites of the TFs by MIL3D. To this end, we ranked all 7-mers in the positive test probe sequences by their predicted probability of binding, and extracted the top ten most frequent 7 -mers that have a predicted binding probability at least 0.85 . Table 2 shows those 7-mers and their frequency. In general, a high total occurrence of these ten 7-mers or the existence of a few dominating 7-mers indicates stronger consensus binding site. Interestingly, the TFs that the simple counting method performed well for, such as Foxo4 and Gmeb2, tend to have a strong consensus motif, while the TFs that the simple counting method failed to model do not seem to have a strong consensus motif. A significant positive correlation (Pearson correlation coefficient $=0.45, p$-value $=0.05$ ) exists between the AUC of 5 -mer counting and the total occurrence of the top-ten 7-mers, while such correlation

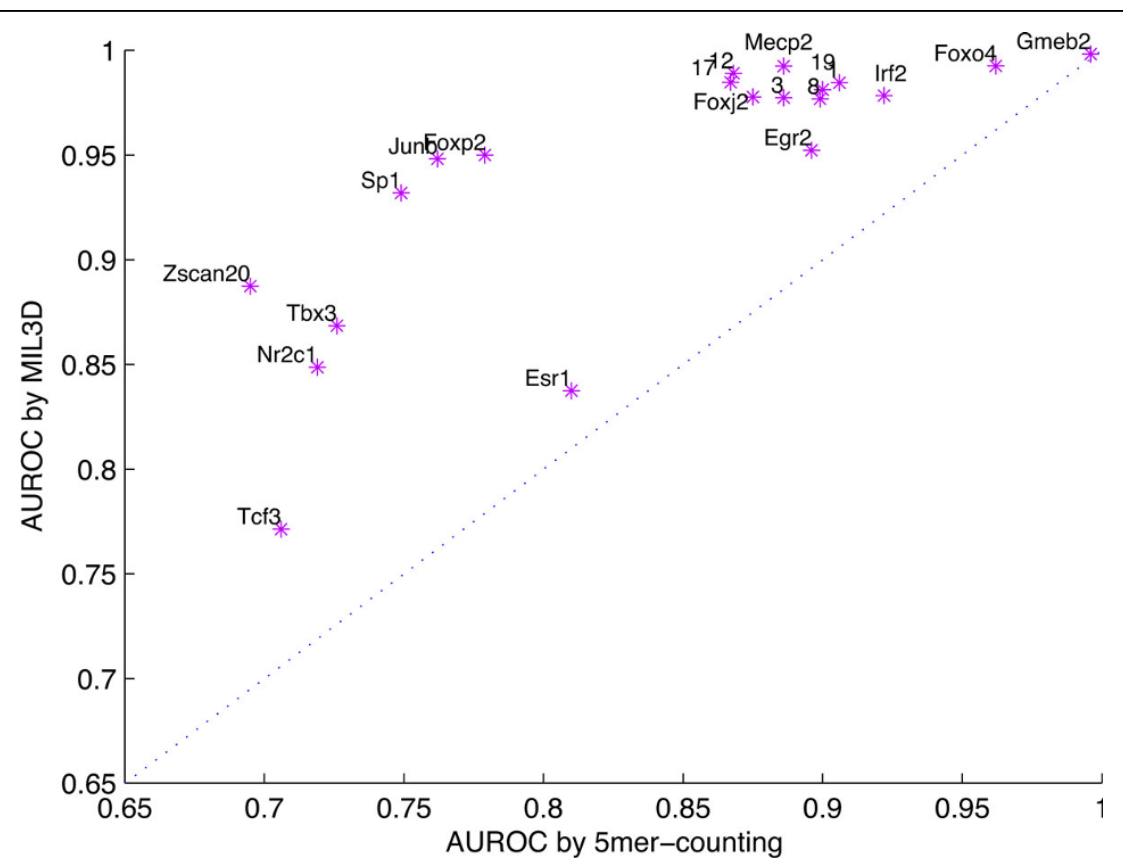

Figure 3 Scatter plot for performance comparison between 5 mer-counting method and MIL3D. The twenty purple stars represent the twenty TFs. The names of the TFs are shown on the upper left corner of each of the corresponding star signs, except for TF $1,3,8,12,17$ and 19. They are shown by numbers to avoid text overlapping. TF 1, 3, 8, 12, 17 and 19 represent Cebpb, Foxo1, Pou3f1, Foxo3, Foxp1 and Sox14 respectively. 
Table 2 Occurrence of ten most frequent high-scoring 7-mers in positive probe sequences for each TF (only 7-mers with a predicted probability of binding $>0.85$ are considered.)

\begin{tabular}{|c|c|c|c|c|c|c|c|c|c|}
\hline Cebpb & & Esr1 & & Foxo1 & & Foxo4 & & Foxp2 & \\
\hline GATTGCA & 48 & AGTCAAG & 9 & ACAAACA & 53 & GATAAAC & 30 & TGTATAC & 32 \\
\hline TGTTGCA & 43 & ATGATCT & 7 & TGTTATT & 52 & AATAAAC & 29 & TGTTGTA & 21 \\
\hline TATTGCG & 42 & ACGTCGA & 6 & TGTIIT & 47 & CTATTTA & 27 & TGTGTAC & 20 \\
\hline CATTGCA & 39 & ACGTTCT & 5 & TGTTGT & 44 & AATAAAT & 23 & CTTGATA & 12 \\
\hline CATTGCG & 38 & AGGTGCA & 4 & ACAACAT & 41 & CATAAAC & 22 & AATATCC & 10 \\
\hline GATTGCG & 35 & ATGTTCT & 4 & TGTTGAT & 41 & GATAAAT & 21 & TGTGCTT & 10 \\
\hline TITGCA & 35 & ACGATAT & 3 & TGTTATC & 32 & CATAAAT & 19 & TCTGTTC & 10 \\
\hline CGTTGCA & 33 & AGTCAGC & 3 & ACAATAA & 31 & CTATTTG & 11 & ACTATCC & 9 \\
\hline TTGCGAA & 26 & ACGCCCT & 2 & AAACAGG & 21 & GTATTTC & 7 & ATTATCC & 9 \\
\hline Total & 339 & Total & 43 & Total & 362 & Total & 189 & Total & 133 \\
\hline Irf2 & & Mecp2 & & Pou3f1 & & Sp1 & & Tcf3 & \\
\hline TTTCATT & 58 & CACACAG & 24 & ТTAАTTA & 62 & TCCGCCC & 38 & CACCTGG & 60 \\
\hline TाTCGAT & 56 & ACACAGG & 24 & СТАATTA & 60 & ACCGCCC & 35 & CACCTGA & 43 \\
\hline TTCGGT & 56 & GACACAG & 17 & ATTAATT & 57 & TGGGCGG & 30 & TCAGCTG & 16 \\
\hline TTCGCT & 54 & ACACAGC & 15 & GTAATTA & 53 & GCCGCCC & 26 & GAATGCA & 13 \\
\hline TाTCACT & 53 & TACACAG & 13 & ATTAATA & 50 & GGGCGGG & 23 & CACCAGG & 8 \\
\hline TाTCAGT & 46 & ACACGCT & 13 & ATTAATG & 46 & TACTCCA & 14 & TCGTCAC & 7 \\
\hline TITTCGT & 46 & ACACGGC & 13 & TATAATT & 43 & TCTGGGC & 11 & CACCAGA & 5 \\
\hline TTTCTAT & 43 & TAAAGTA & 10 & АTTATTA & 42 & TGGGAGG & 11 & GCCAGAA & 3 \\
\hline TाTTCAT & 41 & CACTGAC & 9 & AATAATT & 40 & GGGAGGG & 10 & TCAGCTT & 3 \\
\hline Total & 453 & Total & 138 & Total & 453 & Total & 198 & Total & 158 \\
\hline Egr2 & & Foxo3 & & Gmeb2 & & $\mathrm{Nr} 2 \mathrm{Cl}$ & & Tbx3 & \\
\hline CACCCAC & 60 & GCTGTTT & 22 & GTACGTA & 68 & ATGACCC & 61 & TAGGTGT & 32 \\
\hline CTCCCAC & 56 & CTTATT & 21 & TGACGTA & 65 & GTGACCC & 60 & AAGGTGT & 30 \\
\hline GGCCCAC & 47 & AATATTT & 20 & GACGTAA & 63 & TTGACCC & 60 & CAGGTGT & 23 \\
\hline AGCCCAC & 43 & GTTATTT & 20 & TACGTAC & 63 & TTGACCT & 60 & AGGGTGT & 17 \\
\hline TACCCAC & 42 & CGTATTT & 19 & TTACGTC & 60 & GTGACCT & 57 & TGGGTGT & 16 \\
\hline ATCCCAC & 39 & TCTATTा & 19 & CTACGTA & 59 & ATGACCT & 45 & GAGGTGC & 15 \\
\hline TTCCCAC & 27 & ATTATTा & 18 & TACGTCA & 53 & CTGACCT & 41 & TCATCAC & 13 \\
\hline AACCCAC & 26 & CCTGTT & 17 & CGACGCA & 51 & TGACCCT & 41 & СТCACCT & 12 \\
\hline GTCCCAC & 23 & TATATTT & 17 & TGACGTT & 51 & CTGACCC & 31 & CGGGTGT & 12 \\
\hline Total & 363 & Total & 173 & Total & 533 & Total & 456 & Total & 170 \\
\hline Foxj2 & & Foxp1 & & Junb & & Sox14 & & Zscan20 & \\
\hline ATGTTTA & 48 & CGTAAAC & 32 & TGCCACA & 12 & CAATTCA & 34 & AGGGTTT & 20 \\
\hline AACAAAC & 44 & GGTAAAC & 30 & AGAATTC & 12 & CAATTGA & 30 & AGGGTCG & 16 \\
\hline GTGTTTA & 41 & AGTAAAC & 28 & AATCTIT & 11 & CAATAGT & 26 & AGGGTTG & 14 \\
\hline AACAAAT & 35 & TGTAAAT & 27 & GTCAACA & 10 & CAATAAA & 24 & TACAGGT & 14 \\
\hline TTGTTT & 35 & AGTAAAT & 23 & ACGTTCC & 8 & CAATGTA & 24 & AGGGTCA & 10 \\
\hline TTGTTG & 30 & CGTAAAT & 18 & GTCCGTA & 7 & CAATAAC & 22 & AACTCTG & 10 \\
\hline AACAAAA & 28 & ACTAAAC & 17 & TTGCGCT & 7 & CAATTAC & 22 & AGGGTCT & 9 \\
\hline AACAAAG & 28 & CAACAGG & 15 & CGCCACA & 7 & CAATACT & 21 & GTAAGGT & 9 \\
\hline TACAAAC & 28 & GGTAAAT & 15 & GCCGTAC & 6 & CAATACA & 19 & ACAATAG & 8 \\
\hline Total & 317 & Total & 205 & Total & 80 & Total & 222 & Total & 110 \\
\hline
\end{tabular}

does not exist between the AUC of MIL3D and 7-mer occurrences (Pearson correlation coefficient $=0.27, p$-value $=0.25)$. As shown in Figure 4, the performance gain of MIL3D over 5-mer counting is negatively correlated with 7 -mer occurrences (Pearson correlation coefficient $=-0.42$, $p$-value $=0.07)$. The TF Esr1 seems to lack strong consensus and both MIL3D and 5mer-counting performed relatively poorly. When Esr1 is excluded, the correlation between performance gain by MIL3D over 5-mer counting and 7-mer occurrence becomes much more significant 


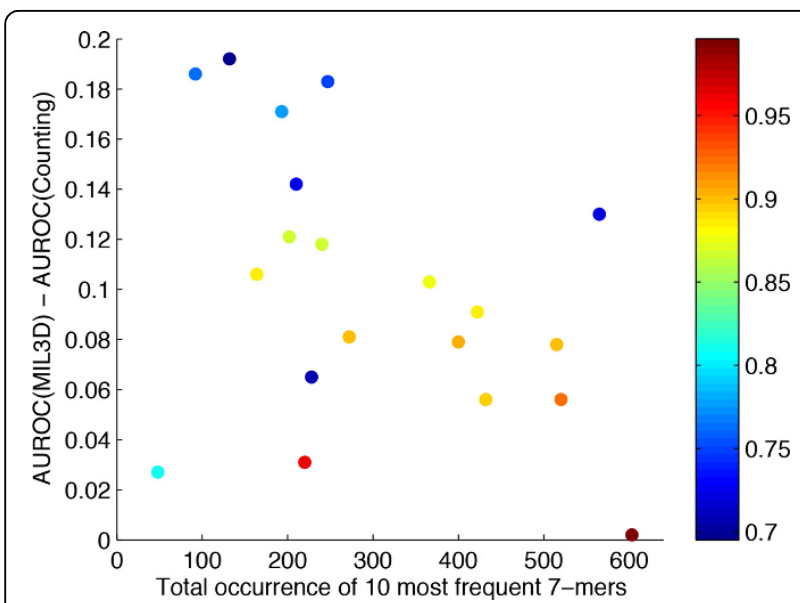

Figure 4 Correlation between 7-mer frequency and performance gain by MIL3D. Not considering TF Esr1 (the light blue, bottom left data point), for which both MIL3D and simple counting had low accuracy, a significant negative correlation (Pearson correlation coefficient $=-0.61, p$-value $=0.006$ ) is observed between the total occurrence of the top-10 7-mers in the test sequences and the performance gain achieved by MIL3D. Color of data points represents the AUC by the simple counting method.

(Pearson correlation coefficient $=-0.61, p$-value $=0.006$ ). This analysis suggests that while a simple counting method works well for TFs that contain a single dominating consensus, the MIL3D approach is more versatile and can utilize subtle structural similarities when a strong sequence consensus is not available.

We also examined the correlation between the performance gain of MIL3D and the GC content of the probe sequences. It is well known that genomic sequences have non-uniform frequency of nucleotides and that certain promoter elements show strong preference for either the $\mathrm{GC}$-rich or the GC-poor core promoters. While the collection of PBM probe sequences may not reflect the same GC content characteristics of real promoters, we observed an interesting, strong negative correlation between GC content of the probes and the performance gain by MIL3D (Figure 5, Pearson correlation coefficient $=-0.44$, $p$-value $=0.05)$; in contrast, the performance of 5 -mer counting method is independent of GC content of the probe sequences (Pearson correlation coefficient $=-0.03$, $p$-value $=0.90)$. Vertebrate promoters are marked by an enrichment of both GC dinucleotide and G/C mononucleotide frequency. Therefore, the improved performance of our method in these relatively GC-poor regions may suggest that overall nucleotide composition rather than particular consensus pattern could have played a significant role in determining the binding affinity of the mouse TFs affected, which is also consistent with the results obtained above in correlation between performance and 7-mer occurrences. It will be interesting to investigate such relationships in multiple other organisms given that

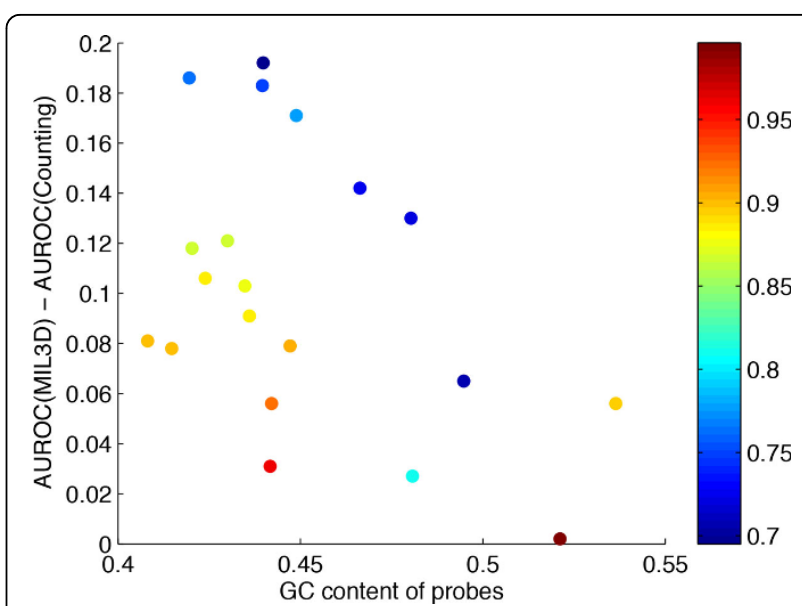

Figure 5 Correlation between probe sequence GC content and performance gain by MIL3D. A significant negative correlation (Pearson correlation coefficient $=-0.44, p$-value $=0.05$ ) is observed between the GC content of the test probe sequence and the performance gain achieved by MIL3D. Color of data points represents the AUC by the simple counting method.

PBM data for those are now available, which may help understand TF-DNA interactions in the context of evolution.

\section{Conclusions}

In this paper, we proposed a TFBS modeling and prediction approach - MIL3D. Combining MIL and structural properties of DNA, it models and predicts in vitro transcription factor bindings more accurately than the widely used $k$-mer counting methods on in vitro protein binding microarray data for twenty mouse transcription factors. Our analysis showed that the MIL3D approach can utilize subtle structural similarities when a strong sequence consensus is not available and demonstrated the encouraging potential of using MIL and structural properties of DNA to study molecular regulatory networks. The key idea in our method can be easily combined with current state-of-the-art $k$-mer based models, which often involve additional normalization, filtering, or transformation of data, to increase the prediction accuracy of TF-DNA interactions.

\section{Competing interests}

The authors declare that they have no competing interests.

\section{Authors' contributions}

ZG proposed the study, performed the data analysis and drafted the manuscript. JR conceived of the study, participated in its design and helped to draft the manuscript. All authors read and approved the final manuscript.

\section{Declarations}

This article was funded by NSF grant IIS-1218201, NIH grants SC3GM086305, U54CA113001, G12MD007591, and a UTSA Tenure-track Research Award. This article has been published as part of BMC Genomics Volume 16 Supplement 4, 2015: Selected articles from the IEEE International Workshop 
on Genomic Signal Processing and Statistics (GENSIPS) 2013. The full contents of the supplement are available online at http://www. biomedcentral.com/bmcgenomics/supplements/16/S4.

\section{Published: 21 April 2015}

\section{References}

1. Berg OG, von Hippel PH: Selection of DNA binding sites by regulatory proteins: Statistical-mechanical theory and application to operators and promoters. Journal of molecular biology 1987, 193(4):723-743.

2. Stormo GD: Consensus patterns in DNA. Methods in enzymology 1990, 183:211-221

3. Zhao $X$, Huang $H$, Speed TP: Finding short DNA motifs using permuted markov models. Journal of Computational Biology 2005, 12(6):894-906

4. Badis G, Berger MF, Philippakis AA, Talukder S, Gehrke AR, Jaeger SA, Chan ET, Metzler G, Vedenko A, Chen X, et al: Diversity and complexity in DNA recognition by transcription factors. Science 2009, 324(5935):1720-1723.

5. Weirauch MT, Cote A, Norel R, Annala M, Zhao Y, Riley TR, Saez-Rodriguez J, Cokelaer T, Vedenko A, Talukder S, et al: Evaluation of methods for modeling transcription factor sequence specificity. Nature biotechnology 2013, 31:126-134.

6. Lei C, Ruan J: A particle swarm optimization-based algorithm for finding gapped motifs. BioData mining 2010, 3:9.

7. Nutiu R, Friedman RC, Luo S, Khrebtukova I, Silva D, Li R, Zhang L, Schroth GP, Burge CB: Direct measurement of DNA affinity landscapes on a high-throughput sequencing instrument. Nature biotechnology 2011, 29(7):659-664.

8. Maerkl SJ, Quake SR: A systems approach to measuring the binding energy landscapes of transcription factors. Science 2007, 315(5809):233-237.

9. Gao Z, Zhao R, Ruan J: A genome-wide cis-regulatory element discovery method based on promoter sequences and gene co-expression networks. BMC genomics 2013, 14(Suppl 1):4.

10. Bauer AL, Hlavacek WS, Unkefer PJ, Mu F: Using sequence-specific chemical and structural properties of DNA to predict transcription factor binding sites. PLOS computational biology 2010, 6(11):1001007.

11. Chen QK, Hertz GZ, Stormo GD: Matrix search 1.0: a computer program that scans DNA sequences for transcriptional elements using a database of weight matrices. Computer applications in the biosciences: CABIOS 1995, 11(5):563-566.

12. Djordjevic M, Sengupta AM, Shraiman BI: A biophysical approach to transcription factor binding site discovery. Genome research 2003, 13(11):2381-2390.

13. Kel AE, Gößling E, Reuter I, Cheremushkin E, Kel-Margoulis OV, Wingender E: Matchtm: a tool for searching transcription factor binding sites in DNA sequences. Nucleic acids research 2003, 31(13):3576-3579.

14. Gordân R, Hartemink AJ, Bulyk ML: Distinguishing direct versus indirect transcription factor-DNA interactions. Genome Research 2009, 19(11):2090-2100.

15. Mukherjee S, Berger MF, Jona G, Wang XS, Muzzey D, Snyder M, Young RA, Bulyk ML: Rapid analysis of the DNA-binding specificities of transcription factors with DNA microarrays. Nature genetics 2004, 36(12):1331-1339.

16. Maron O, Lozano-Pérez T: A framework for multiple-instance learning. Advances in neural information processing systems 1998, 570-576.

17. Andrews S, Tsochantaridis I, Hofmann T: Support vector machines for multiple-instance learning. Advances in Neural Information Processing Systems 2002, 561-568.

18. Auer P: On learning from multi-instance examples: Empirical evaluation of a theoretical approach. In Proc. 17th International Con. on Machine Learning. Volume 97. Morgan Kaufmann; 1997:21-29.

19. Wang J, Zucker J-D: Solving the multiple-instance problem: A lazy learning approach. Proc. 17th International Con. on Machine Learning Morgan Kaufman; 2000, 1119-1125.

20. Stolovitzky G, Monroe D, Califano A: Dialogue on reverse-engineering assessment and methods. Annals of the New York Academy of Sciences 2007, 1115(1):1-22

21. Frank $E, X u X$ : Applying propositional learning algorithms to multiinstance data. Department of Computer Science, University of Waikato, Hamilton, NZ 2003.
22. Hall M, Frank E, Holmes G, Pfahringer B, Reutemann P, Witten IH: The weka data mining software: an update. ACM SIGKDD explorations newsletter 2009, 11(1):10-18.

23. Cheatham TE III: Simulation and modeling of nucleic acid structure, dynamics and interactions. Current opinion in structural biology 2004, 14(3):360-367.

24. Olson WK, Bansal M, Burley SK, Dickerson RE, Gerstein M, Harvey SC, Heinemann U, Lu X-J, Neidle S, Shakked Z, et al: A standard reference frame for the description of nucleic acid base-pair geometry. Journal of molecular biology 2001, 313(1):229-237.

25. Wang W-M, Wu S-Y, Lee A-Y, Chiang C-M: Binding site specificity and factor redundancy in activator protein-1-driven human papillomavirus chromatin-dependent transcription. Journal of Biological Chemistry 2011, 286(47):40974-40986.

26. Li M, Ge Q, Wang W, Wang J, Lu Z: c-jun binding site identification in k562 cells. Journal of Genetics and Genomics 2011, 38(6):235-242.

doi:10.1186/1471-2164-16-S4-S3

Cite this article as: Gao and Ruan: A structure-based Multiple-Instance Learning approach to predicting in vitro transcription factor-DNA interaction. BMC Genomics 2015 16(Suppl 4):S3.

\section{Submit your next manuscript to BioMed Central and take full advantage of:}

- Convenient online submission

- Thorough peer review

- No space constraints or color figure charges

- Immediate publication on acceptance

- Inclusion in PubMed, CAS, Scopus and Google Scholar

- Research which is freely available for redistribution
Ciomed Central 